\title{
Combined acute interstitial pneumonitis and pancytopenia induced by low-dose methotrexate in a hemodialysis patient treated for bullous pemphigoid*
}

\author{
Haibo Liu ${ }^{1}$ \\ Wenliang Yan $^{1}$
}

\author{
Fang $\mathrm{Liu}^{1}$ \\ Hong Sang ${ }^{1}$
}

Min Zhang ${ }^{1}$

DOI: http:/ /dx.doi.org/10.1590/abd1806-4841.20153692

\begin{abstract}
A bstract: Methotrexate has been widely used for many years in the treatment of a variety of diseases. Acute pneumonitis and bone marrow suppression are very serious side effects in methotrexate treatment. A 48-year-old man with end-stage renal disease undergoing chronic hemodialysis developed combined acute pneumonitis and pancytopenia after a cumulative dose of $20 \mathrm{mg}$ methotrexate for bullous pemphigoid. Continuous renal replacement therapy (CRRT) can efficiently decrease serum methotrexate concentration. A rapid improvement of clinical symptoms and resolution of pulmonary opacification were found after CRRT. Blood cell counts returned to normal after component blood transfusion and cytokine supportive therapy. Patients with impaired renal function are at high risk of methotrexate toxicity, and low-dose methotrexate should be prescribed with great caution.
\end{abstract}

Keywords: Methotrexate; Pancytopenia; Pneumonia; Renal dialysis

\section{INTRODUCTION}

Methotrexate (MTX) is always taken on a once-weekly basis, with smaller doses for skin problems. Along with its needed effects, MTX has several potential side effects, including acute pneumonitis and bone marrow suppression, which are very serious and sometimes life-threatening. The background dermatological diseases of MTX pneumonitis reported to date include psoriasis, mycosis fungoides, and pemphigus vulgaris. ${ }^{1}$ No MTX pneumonitis has been found in patients with bullous pemphigoid, and only one case of fatal pancytopenia has been reported in a hemodialysis patient with bullous pemphigoid. ${ }^{2}$ Here we report a case of combined acute intersitial pneumonitis and pancytopenia induced by low-dose MTX in a hemodialysis patient treated for bullous pemphigoid.

\section{CASE REPORT}

A 48-year-old man was treated with hemodialysis (three times/week) in 2008 for end-stage renal disease due to chronic glomerulonephritis. On $26^{\text {th }}$ June 2013, the patient was hospitalized because of bullous pemphigoid, which was diagnosed on clinical, histopathological, and immunofluorescence grounds (Figure 1A). Histopathological examination showed subepidermal blister formation and direct immunofluorescence revealed a linear deposit of IgG and C3 (data not shown) along the basement membrane (Fi-gures $1 \mathrm{~B}$ and $1 \mathrm{C})$. Application of methylprednisolone (60 mg daily) and topical halometasone failed to control the disease. A heterozygous (CT), methylenetetrahydrofolate reductase (MTHFR) genotype was confirmed by PCR. Oral MTX (10 mg weekly) was then given and folic acid was prescribed. MTX addition led to rapid symptomatic improvement and cessation of new bullae formation. On the fifth day, after the second MTX dose, the patient complained of acute shortness of breath, non-productive cough, and fever. Physical examination revealed inspiratory rales over both lower lung fields. Arterial blood gas analysis revealed a $\mathrm{PaO} 2$ of $49 \mathrm{~mm} \mathrm{Hg}, \mathrm{PaCO}_{2}$ of $30 \mathrm{~mm} \mathrm{Hg}$, and $\mathrm{pH}$ of $7.42 \mathrm{on}$ room air. A high-resolution $\mathrm{CT}$ scan showed extensive ground-glass infiltrates in both lungs (Figure 2A). The

Received on 10.05.2014

Approved by the Advisory Board and accepted for publication on 09.06.2014

Study conducted at the Department of Dermatology, Jinling Hospital, affiliated to the Nanjing University, School of Medicine - Nanjing, China.

Financial Support: None.

Conflict of Interest: None.

Nanjing University - Nanjing, China.

(C)2015 by Anais Brasileiros de Dermatologia 

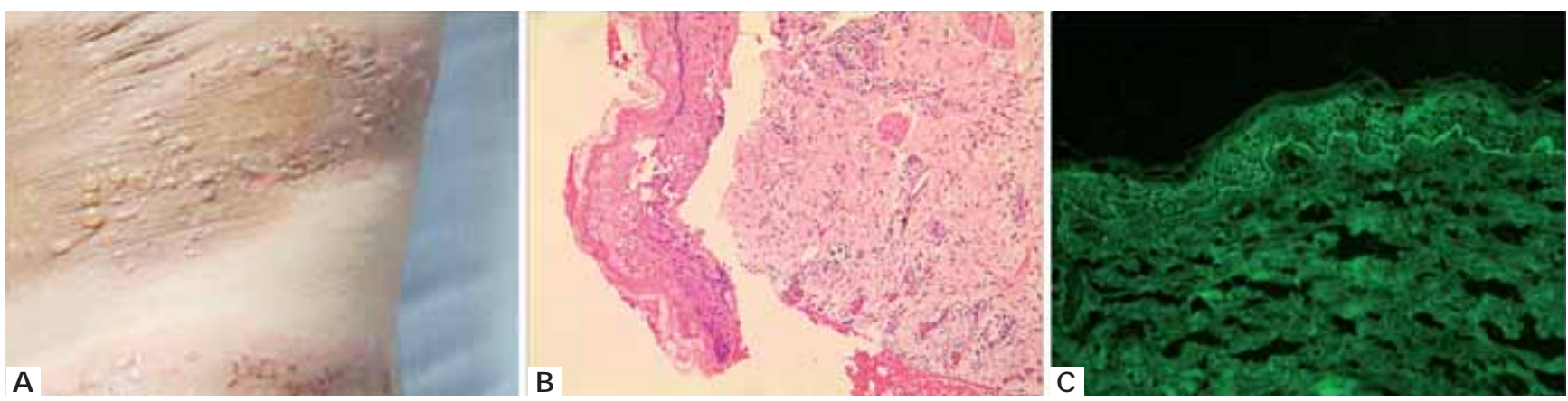

Figure 1: A. Blisters on the trunk of the patient's body. B. Histopathological examination shows subepidermal blister formation and perivascular infiltrates in the dermis. C. Direct immunofluorescence reveals a linear deposit of IgG along the basement membrane
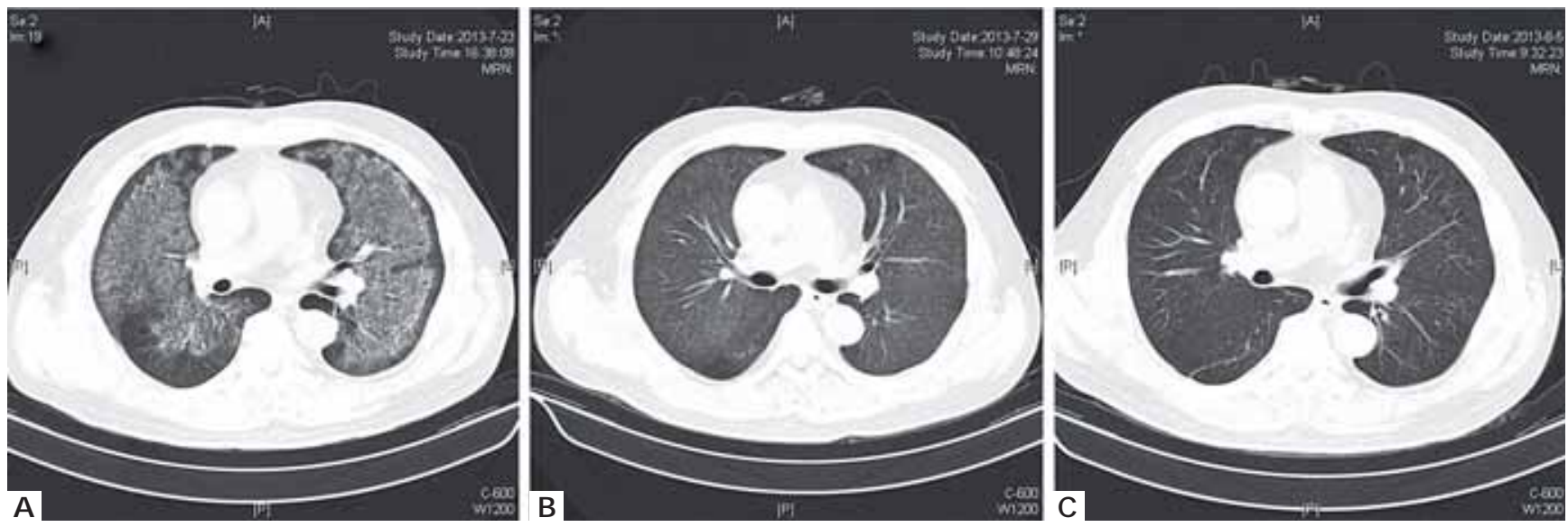

Figure 2: A.CT scan shows extensive ground-glass infiltrates in both lungs. B and C. The resolution of pulmonary opacification during (B) and after (C) CRRT therapy

patient satisfied criteria for MTX pneumonitis according to the aforementioned findings and other supportive evidences. ${ }^{1,3}$ MTX was stopped, methylprednisolone was maintained at $60 \mathrm{mg}$ daily, and the patient was treated with nasal oxygen. The hemodialysis was then carried out once daily to eliminate MTX. Serum MTX level was measured by using the Syva enzyme multiplied immunoassay technique (EMIT). After five hemodialysis procedures, the serum MTX level was still $0.14 \mu \mathrm{mol} / \mathrm{L}$, the same as after the first hemodialysis. No apparent improvement in pulmonary infiltrates was found, as evidenced by repeat CT scan. The patient was transferred to the Urology Department to receive continuous renal replacement therapy (CRRT). After one day of CRRT using continuous veno-venous haemofiltration $(\mathrm{CVVH})$, the patient rapidly improved and serum MTX level decreased from 0.14 (72-hour MTX concentration $>0.1 \mu \mathrm{mol} / \mathrm{L}$ is considered as at risk of toxicity) to $0.07 \mu \mathrm{mol} / \mathrm{L}$. Pulmonary interstitial infiltrates gradually resolved and a nearly complete resolution of pulmonar infiltrates was found 3 days after CRRT initiation. Arterial blood gas analysis was also found to be normal (Figures 2B and 2C). On the sixth day, after the second MTX dose, blood analysis confirmed pancytopenia: WBC count was $1.8^{\prime} 10^{9} / \mathrm{L}$, hemoglobin concentration was $51 \mathrm{~g} / \mathrm{L}$, and platelet count was $49^{\prime} 10^{\circ} / \mathrm{L}$. His pancytopenia recovered after component blood transfusion and cytokine supportive treatment (G-CSF and IL-11). Methylprednisolone was converted to prednisone to control skin lesions after recovery from pneumonitis. The patient did not complain of respiratory symptoms during a 3-month follow-up.

\section{DISAUSSION}

MTX is not recommended in case of a creatinine clearance $<10 \mathrm{~mL} / \mathrm{mn}^{2}$. A study has suggested that MTX, which is highly bound to albumin, can be removed by hemodialysis. ${ }^{4}$ This finding makes MTX a viable treatment option in uremic patients. Recently, Vilay et al have demonstrated successful treatment of MTX intoxication by using continuous extracorporeal therapy and glucarpidase in a patient with osteosarcoma. ${ }^{5}$ Of particular importance is the use of continuous veno-venous hemodialysis (CVVHD) and continuous veno-venous hemodiafiltration (CVVHDF). CVVHD and CVVHDF are different CRRT modes and either one of them can efficiently increase MTX removal. This 
view is also supported in our patient, who received CRRT therapy using CVVH.

Another report has stated MTX is poorly removed by hemodialysis, although removal through hemodialysis is greater than through peritoneal dialysis. 4,6 The authors believe that MTX, even in low dose, should not be prescribed to dialysis patients. ${ }^{6}$ Our findings also suggested poor removal of MTX by hemodialysis, since five hemodialysis procedures failed to reduce serum MTX. The failure to eliminate MTX may be associated with the absence of high-flux membrane in hemodialysis, as high-flux hemodialysis has been proven to efficiently reduce serum MTX level, despite the failed clearance of polyglutamated MTX metabolite within cells. ${ }^{7}$

\section{REFERENCES}

1. Imokawa S, Colby TV, Leslie KO, Helmers RA. Methotrexate pneumonitis: review of the literature and histopathological findings in nine patients. Eur Respir J. 2000;15:373-81.

2. Seneschal J, Héliot-Hostein I, Taieb A. Pancytopenia induced by low-dose methotrexate in a haemodialysis patient treated for bullous pemphigoid. $\mathrm{J}$ Eur Acad Dermatol Venereol. 2007;21:1135-6.

3. Searles G, McKendry RJ. Methotrexate pneumonitis in rheumatoid arthritis: potential risk factors. Four case reports and a review of the literature. J Rheumatol. 1987;14:1164-71

4. Diskin CJ, Stokes TJ, Dansby LM, Radcliff L, Carter TB. Removal of methotrexate by peritoneal dialysis and hemodialysis in a single patient with end-stage renal disease. Am J Med Sci. 2006;332:156-8.

5. Vilay AM, Mueller BA, Haines H, Alten JA, Askenazi DJ. Treatment of methotrexate intoxication with various modalities of continuous extracorporeal therapy and glucarpidase. Pharmacotherapy. 2010;30:111.

6. Basile C, Montanaro A, Semeraro A. Should low-dose methotrexate therapy be prescribed to dialysis patients? Nephrol Dial Transplant. 2002;17:530-1.

7. Wall SM, Johansen MJ, Molony DA, DuBose TD Jr, Jaffe N, Madden T. Effective clearance of methotrexate using high-flux hemodialysis membranes. Am J Kidney Dis. 1996;28:846-54

8. Salaffi F, Manganelli P, Carotti M, Subiaco S, Lamanna G, Cervini C. Methotrexateinduced pneumonitis in patients with rheumatoid arthritis and psoriatic arthritis: report of five cases and review of the literature. Clin Rheumatol. 1997;16:296-304.

9. Yang CP, Kuo MC, Guh JY, Chen HC. Pancytopenia after low dose methotrexate therapy in a hemodialysis patient: case report and review of literature. Ren Fail. 2006;28:95-7.

10. Toffoli G, Russo A, Innocenti F, Corona G, Tumolo S, Sartor F, et al. Effect of methylenetetrahydrofolate reductase $677 \mathrm{C}-->\mathrm{T}$ polymorphism on toxicity and homocysteine plasma level after chronic methotrexate treatment of ovarian cancer patients. Int J Cancer. 2003;103:294-9.
The risk factors that have been suggested for MTX pneumonitis include preexisting pulmonary disease or abnormalities on chest radiographs, smoking, acetylsalicylic acid use, and renal insufficiency. ${ }^{8}$ Risk factors for pancytopenia include impaired renal function, hypoalbuminemia, advanced age, concurrent infection, and/or concurrent medication. ${ }^{9}$ As to our patient, impaired renal function may be the most important risk factor both for MTX pneumonitis and pancytopenia. It is uncertain whether the heterozygous M TH FR genotype in our patient is associated with MTX toxicity, because CC and CT genotypes showed no significant differences in risk of MTX toxicity. ${ }^{10}$

Low-dose MTX must be prescribed with great caution to uremic patients. Alternative medicines (azathioprine or mycophenolate mofetil) should be considered.]

\author{
M AILING ADDRESS: \\ $H$ ong Sang \\ 305 East Zhongshan Road, \\ $\mathrm{N}$ anjing \\ 210002, China. \\ E-mail: shzwqzsl@163.com
}

H ow to cite this article: Liu H, Liu F, Zhang M, Yan W, Sang H. Combined acute interstitial pneumonitis and pancytopenia induced by low-dose methotrexate in a hemodialysis patient treated for bullous pemphigoid. An Bras Dermatol. 2015;90 (3 Suppl 1): S43-5. 